\title{
Feeling free: external influences on endogenous behavior
}

\begin{tabular}{|c|c|}
\hline Journal: & Quarterly Journal of Experimental Psychology \\
\hline Manuscript ID & QJE-STD-18-068.R3 \\
\hline Manuscript Type: & Standard Article \\
\hline $\begin{array}{r}\text { Date Submitted by the } \\
\text { Author: }\end{array}$ & $\mathrm{n} / \mathrm{a}$ \\
\hline Complete List of Authors: & $\begin{array}{l}\text { Charles, Lucie; University College London Institute of Cognitive } \\
\text { Neuroscience, } \\
\text { Haggard, Patrick; University College London Institute of Cognitive } \\
\text { Neuroscience }\end{array}$ \\
\hline Keywords: & Action, Decision-making, Freedom of Choice, Volition \\
\hline
\end{tabular}




\section{Title: Feeling free: external influences on endogenous behavior}

2

3 Authors: Lucie CHARLES ${ }^{a^{*}}$, Patrick HAGGARD a

4

5 Affiliations: ${ }^{a}$ Institute of Cognitive Neuroscience, University College London, London WC1N 3AR

6 United Kingdom

7

8 Corresponding author: Lucie Charles

9 Institute of Cognitive Neuroscience

10 Alexandra House, 17 Queen Square

11 WC1N3AR London

12 Tel: +441865271302

13 lucie.charles.ens@googlemail.com

16 Number of pages: 23

17 Number of figures: 5

18 Number of words for abstract: $\mathbf{2 4 2}$

19 Number of words for Introduction: $\mathbf{7 5 0}$

20 Number of words for Materials\& Methods: 1248

21 Number of words for Results: 1614

22 Number of words for Discussion: $\mathbf{1 3 0 3}$ 
24 ABSTRACT (242)

When we are presented with two equally appealing options, how does the brain break the symmetry between them and make a choice? Recent research has proposed that when no clear information can guide decisions, we use irrelevant noise to tip the scale in favor of one alternative and decide how to act. In the present study, we investigated this issue exploring how human decisions were influenced by noise in a visual signal that cued instructed or free choice. Participants were presented with random-dot kinematograms, moving unidirectionally either upward or downward (in instructed trials) or both upward and downward simultaneously (free-choice trials). By varying the coherence of dot motion, we were able to test how moment-to-moment fluctuations in motion energy could influence action selection processes. We also measured participants' awareness of such influence. Our results revealed three novel findings: Participants' choices tended to follow fluctuations in dot motion, showing that sensory noise biased "free" selection between actions, irrespective of the clarity of the free cue. However, participants appeared to remain unaware of that influence, since subjective ratings of freedom did not correlate with the degree of sensory biasing. In one exception to this general rule, we found that, when participants resisted the bias and made a choice opposite to the one suggested by the stimulus, they reported strong subjective sense of having chosen independently of the stimulation. This result suggests that inhibitory control is tightly linked to the sense of freedom of choice.

Keywords: Action, Decision-making, Freedom of Choice, Volition 


\section{INTRODUCTION (750 WORDS)}

The question of what constitutes a free choice, and of whether any action is indeed truly free, has been a matter of intense debate for many years (Libet et al., 1983; Plato, 1987; Wegner, 2004). Indeed, free decisions constitute an empirical challenge: it remains difficult to understand how we can choose an action in the absence of external sensory signals or preference favoring it.

Recent research developed cognitive models explaining how people may make decisions in such contexts and produce self-generated actions (Nachev, 2010; Nachev et al., 2005; Nachev and Husain, 2010; Passingham et al., 2010; Schüür and Haggard, 2011) independently of prior sensory cues (Bode et al., 2013; Hoffstaedter et al., 2013; Soon et al., 2008, 2013; Wisniewski et al., 2016). In the absence of external stimuli, irrelevant internal noise could be accumulated, rather than the familiar accumulation of external sensory evidence, to break the symmetry between different action options (Murakami et al., 2014; Schurger et al., 2012). This model has been proven powerful in predicting the onset of self-initiated action and seems to find support in neural responses accompanying free choices (Bode et al., 2013; Murakami et al., 2014; Schurger et al., 2012), highlighting the similarity between perceptual decision in high-uncertainty conditions and free decisions (Bode et al., 2013).

However, the nature of these putative internal noise sources influencing decisions is unclear. Previous research has focused on trying to remove any factors that could bias self-initiated actions (Bode et al., 2011, 2013, 2014; Lages and Jaworska, 2012; Mattler and Palmer, 2012; Schultze-Kraft et al., 2016; Schurger et al., 2012; Soon et al., 2008, 2013; Wisniewski et al., 2016), by using obviously ambiguous signals such as double-pointing arrows to cue free trials (Kiesel et al., 2006; Le Bars et al., 2016; Wenke et al., 2010). We suggest an alternative approach, namely evaluating whether random fluctuations present in afferent signals reaching the brain are used to break the symmetry between different options for endogenous action. We investigated how much participants were influenced by exogenous perceptual signals when making free decisions, and how much they were aware of that influence. This approach allows us to determine the cognitive strategies developed to make free choices and tackle the novel question of whether we are conscious of the extent to which our decisions are truly endogenous, or based on afferent information.

Similar to classic studies investigating free actions (Kiesel et al., 2006; Le Bars et al., 2016; Wenke et al., 2010), we contrasted trials in which the required action was guided by an unambiguous sensory signal to trials in which intrinsically ambiguous sensory information required participants to make free decisions. In place of the classic single vs double-pointing arrows used in many free-choice studies (Eimer and Schlaghecken, 1998) however, we used random-dot motion stimuli. In instructed trials, the dots moved either up or down, and the participants responded by pressing the corresponding arrow key. In free-choice trials however, the direction of the motion was ambiguous, with dots moving equally in both directions. Participants were then free to press either of the two keys. We varied this mean motion 
78 coherence (i.e., the percentage of dots moving in the same direction) to elicit distinct difficulty 79 conditions: in the easy condition, participants could readily recognize when motion stimuli were 80 bidirectional, and they therefore had to choose freely. In contrast, in the hard condition, motion 81 coherence was set too low to distinguish bidirectional from unidirectional motion, leaving uncertainty 82 about whether they had to act endogenously or exogenously. After each choice, participants rated their 83 subjective sense of freedom of choice over their decision, indicating whether they felt their choice was 84 driven by the sensory information or whether they made up their mind about which key to press 85 independently of what they saw on the screen. The motion energy of random-dot motion stimuli 86 fluctuates around its mean on a moment-to-moment basis. By retrieving on a trial-by-trial basis how 87 much the stochastic fluctuations in sensory information favored the choice made by the participants, we 88 were able to study how much free and instructed actions were driven by external sensory information, 89 and whether this affected the subjective feeling of freedom of choice. In particular, we aimed to test 90 whether the subjective ratings of freedom of choice predicted the objective level of entrainment by the 91 stimulus. Such a result would indicate good introspective abilities regarding actual freedom of 92 choice. Further, by contrasting easy and hard trials, we could determine whether the ambiguity 93 regarding the type of decision required influenced these introspective processes. 


\section{Task}

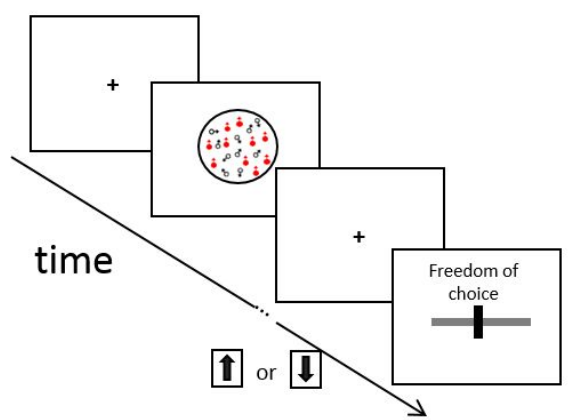

1. Perceptual decision (up or down)

2. Freedom of choice rating

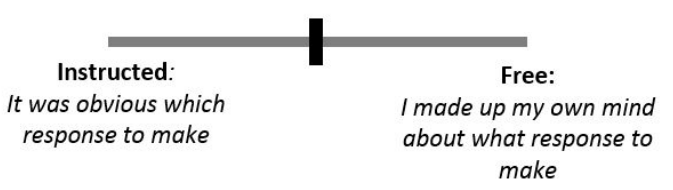

\section{Stimuli}

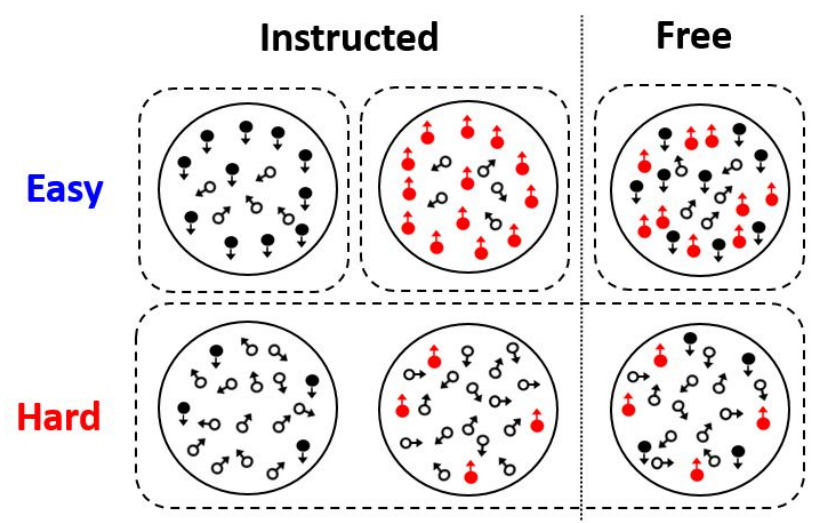

94

Figure 1: Experimental procedure. During the main task, participants were presented with random-dot stimuli moving in either upward or downward directions (instructed trials) or in a mix of both upward and downward directions (free trials). Participants had to respond up or down when the motion was unidirectional (instructed trials), while they were free to press either key when the motion was bidirectional (free trials). Two conditions of difficulty were randomly intermixed. In the Easy condition, motion coherence was high, so upward, downward and bidirectional stimuli were clearly identifiable. In the Hard condition, low motion coherence made upward, downward and bidirectional trials all appear similar. At the end of the trial, participants rated their subjective sense of freedom of choice with a continuous scale. 


\section{MATERIAL \& METHODS (1248 WORDS)}

\subsection{Participants}

Twenty right-handed participants, with normal or corrected-to-normal vision gave informed written consent to participate in a two-session experiment. For 6 participants, the post-test revealed that they had either higher than expected (1 participant) or lower than expected (5 participants) performance in the easy condition respectively (see Supplementary Results 2.1). These participants were therefore excluded of further analysis. Fourteen participants ( 8 females, mean age 22 years) were therefore included in the final sample. This study was approved by the UCL Research Ethics Committee (ICN-PH-PWB-20-02-2014c).

\subsection{Stimuli}

Stimuli consisted of random-dot kinematograms (RDK) appearing in a $5^{\circ}$-diameter aperture presented on a computer monitor with a frame rate of $60 \mathrm{~Hz}$ (see Supplementary Methods 1.1). Importantly each RDK was composed of two clouds of dots moving either in the same direction (unidirectional motion toward up or down direction) or in opposite direction (bidirectional motion mixing up and down motion) indicating respectively whether the trial was Instructed or Free. Stimuli were presented on a 17" laptop with a $60 \mathrm{~Hz}$ refresh rate using the MATLAB toolbox Psychtoolbox3.

\subsection{Procedure}

The experiment consisted of three distinct parts: a pre-test, the main experiment, and a post-test and was conducted in two sessions of 80-90 minutes. During session one, participants performed the pre-test ( 25 minutes), then 7 blocks of 96 trials of the main experiment followed by the post-test (5 minutes). During session 2, they performed 9 blocks of the main experiment followed by the post-test.

The goal of the pre-test phase was to establish for each participant the coherence values for random-dot stimuli that brought the participant close to chance in the Hard condition, and that brought them significantly above chance in the Easy condition. These values were then used to produce participant-specific bidirectional stimuli cueing free choices in both the Hard and the Easy condition (see Supplementary Methods 1.2). As can be seen in Figure S1A, our manipulation was successful in finding these threshold levels of coherence. The goal of the post-test was to determine whether at the end of the experiment, participants were still at chance to discriminate a bidirectional motion from the unidirectional motion in the Hard condition, and whether they were still above chance in Easy conditions. We verified in a post-test session at the end of each main experiment session, that these difficulty levels yielded the expected levels of accuracy at the end 
135 of the experiment as well as at the beginning. We excluded participants for which this was not the case (see Supplementary Results 1.1 and Supplementary Results 2.1). started with a small increase of the fixation cross (thickness of the lines doubled) for $50 \mathrm{~ms}$ and after an additional random time variable between 0 and $500 \mathrm{~ms}$, the RDK were displayed. Easy and hard trials were randomly intermixed, using the two respective stimulus coherence levels determined during the pre-test session. Furthermore, two types of motion were randomly presented. In half of the trials, motion was unidirectional, going either in the upward or downward direction in a randomized manner across trials. In the remaining half of the trials the motion was bidirectional. Participants were instructed to respond to the direction of the motion, responding with the up and down arrow keys if they thought the direction of the motion pointed respectively to the upward and downward directions. Additionally, participants were told that if the stimulus was bidirectional, there was no correct response and they were free to press any of the two buttons they wanted, without pressing systematically the same button or using a pre-established sequence of responses. Participants were informed that there would be as many bidirectional as unidirectional trials. The task was unspeeded and stimuli remained on the screen until participant's response.

After their choice, participants were asked to rate their sense of Freedom of Choice (FoC) accompanying their decision. They were instructed to report how much they felt their response was guided by the stimulus they saw on the screen rather than endogenously generated. To do so, a 51-point scale appeared on the screen, with the sentences "It was obvious which response to make" corresponding to the minimum of freedom of choice (scored as 0 ) and "I had to make up my own mind" corresponding to the maximum freedom of choice (scored as 100). Participants moved a cursor (appearing at an initial random location) with the left and right arrow keys and registered their response with the space-bar. Participants were instructed to use the entire range of the scale rather than only the extreme values and that their response should be guided by how they came up with their response on that given trials rather than identification of the free or instructed cue.

Participants performed a total of 16 blocks of 96 trials across the two sessions allowing to obtain a total of 1536 trials in the whole experiment. Each session lasted approximately $1.5 \mathrm{hr}$ and participants usually performed the two sessions on two consecutive days.

\subsection{Motion Energy Analysis}

In order to determine how free choices and subjective sense of freedom of choice were influenced by fluctuation in stimulus strength, we retrieved for each trial the moment-to-moment fluctuation in motion energy, estimating the strength in the stimulus favoring the one direction of motion over time. 
To do so, we use spatial filter applied on the trial frame-to-frame images of the dot-motion stimuli (see Supplementary Methods 1.4). This analysis allowed us to obtain the time-course of fluctuation of the motion energy for each trial (see Figure 2A for illustrative trials). In free trials, the bidirectional motion energy would always average to zero as upward and downward motion would cancel out. However, fluctuations around the zero mean would remain, corresponding to transient up and down motion energy changes. As motion coherence (proportion of dots moving coherently in the same direction) in the easy and hard condition was adjusted for each participant, the obtained timecourses varied greatly between conditions and participants. Therefore, to compare the impact of sensory fluctuations on response choice across conditions and across participants in a meaningful way, the timecourses were normalized (Figure 2B, Supplementary Methods) for each direction of motion, each condition (easy and hard) and each participant.

We then used the reverse correlation method to determine how these fluctuations in motion energy influenced the choice made by the participants (Figure 2D). To do so, for each trial we adjusted the sign of the obtained z-scored time-course according to the participant's response on that trial, so that positive values indicated motion consistent with the choice made, rather than upward or downward motion. Then, we computed for every trial the cumulative sum (or Area Under-Curve, AUC) of the full time-course (Figure 2C, see Figure S1 for AUC values before normalization). The rational of this analysis is that if the fluctuation in the sensory cue do not influence choice, the mean of the time-course and therefore the resulting AUC should average out to zero. However, if the participant is more likely to make a choice consistent with the direction of the fluctuation in the signal, the AUC value across the time-course should be positive. This measure therefore allowed us to estimate whether the fluctuation in motion strength indeed favored the motion reported by the participant or not and to what extent. The obtained trial-by-trial AUC values were then averaged separately for each condition in each participant and analyzed with paired t-tests (two-tailed, unless specified otherwise), repeated measure ANOVA and individual-subject regressions. 

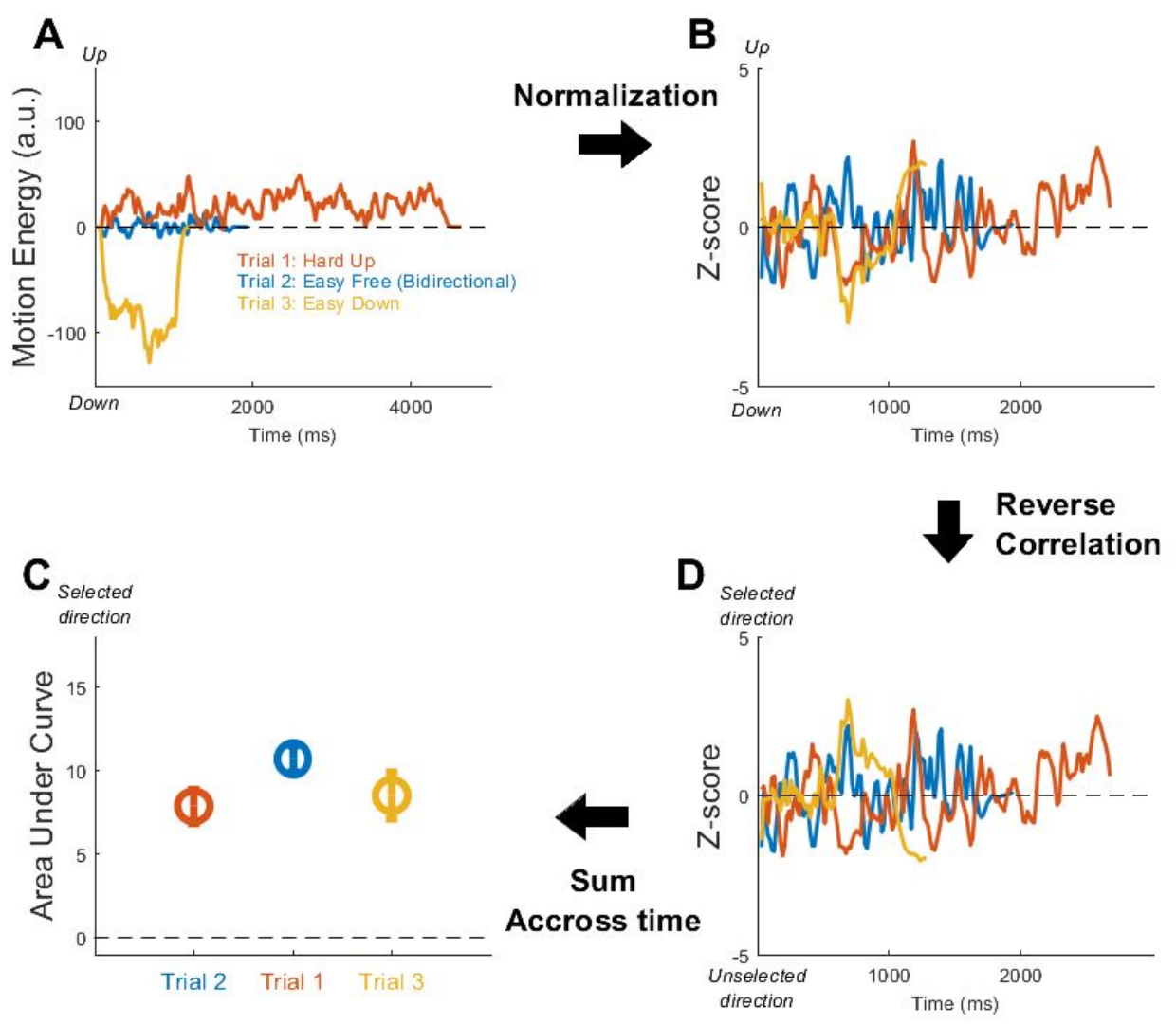

Figure 2: Method used to compute the objective freedom from stimulus-determination. A: Motion 196 energy was retrieved for each trial, as shown for three example trials with different motion direction, coherence (difficulty) and trial-type. B: Each time-course was then normalized by subtracting the mean and dividing by the standard deviation of the time-course over all trials of that condition. C: Each timecourse was then reverse-correlated by assigning it a sign depending on the choice made by the participant so that positive signal corresponded to the direction selected on that trial. D: Area-Under (AUC) curve over the whole trial duration was then computed, providing a measure of how much 203 stochastic fluctuations of the stimulus were associated with the choice made by the participant on that trial. 


\section{RESULTS (1614 WORDS)}

\subsection{Accuracy in pre-test, main experiment and post-test}

Accuracy was computed for easy and hard Instructed trials. As expected, the proportion of correct responses in easy instructed trials was above chance level (Figure $\mathrm{S} 1 \mathrm{~B} \mathrm{t}(13)=\mathbf{1 2 . 6 , p}<.001$, $d=3.37$ ) reaching an average of $90 \%$ across participants. In contrast, hard instructed trials resulted in significantly lower performance $(t(13)=11.9, p<.001, d=3.17$ ) reaching only $\sim 60 \%$ accurate. These results suggest that our staircase procedure was successful in finding thresholds such that participants could clearly distinguish trials as free or instructed in the easy but not in the hard condition. In Free trials, the stimulus motion was bidirectional, so, by definition, either response was considered correct. Nonetheless, we could explore the pattern of responses in free trials. We found that both for easy or hard trials, participants responded with equal probability with their left and right hands (supplementary results 2.3). However, we found that participants' choices were biased by the action made on the previous trial when no evidence was present to guide the current choice (see supplementary results 2.3 ). This could occur either because the stimulus was particularly unclear (Hard condition) or because participants had to make several successive free choices (two consecutive Free trials).

Next we investigated what level of freedom participants felt they had over their choice in each of these conditions, whether their response was indeed influence by the fluctuation in motion energy, and whether objective sensory biasing and subjective freedom of choice correlated with each other.

\subsection{Effect of trial type and difficulty on subjective Freedom of choice}

We investigated how trial difficulty and trial type (Free vs Instructed) influenced the subjective ratings of freedom of choice. Note that participants were instructed not to rate their freedom of choice based on the trial category (free vs instructed) but rather to estimate on each trial their sense that their choice was driven by the presented stimulus or on the contrary that they choose which button to press independently from it.

Easy instructed trials were associated with the lowest freedom of choice ratings (Figure3A,C). In particular, in the easy condition, participants reported significantly less freedom of choice for instructed than free trials (Figure 3A blue line, $\mathbf{t}(\mathbf{1 3})=-4.23, \mathrm{p}<. \mathbf{0 0 1}, \mathbf{d}=\mathbf{- 1 . 1 3}$ ). Interestingly, a similar effect was observed in the hard condition (lower freedom of choice ratings for instructed than free trials $t(13)$ $=\mathbf{2 . 7 3}, \mathrm{p}=\mathbf{. 0 1 7}, \mathrm{d}=\mathbf{- 0 . 7 3}$ ), although to a significantly reduced extent (interaction $\mathrm{F}(\mathbf{1}, 13)=\mathbf{1 4 . 8}, \mathrm{p}=$ 
signals slightly more for instructed than free trials, even when the perceptual information about whether the trial was instructed or free was strongly reduced.

Importantly, no significant difference in freedom of choice ratings was observed between Easy and Hard free-choice trials $(\mathrm{t}(\mathbf{1 3})=\mathbf{- 1 . 6 4}, \mathrm{p}=\mathbf{. 1 3}, \mathrm{d}=\mathbf{- 0 . 4 4 )}$. This suggests that decisions following a stimulus clearly perceived as a free cue (easy condition) were experienced to be just as free as decisions following an unclear stimulus (hard condition).

Interestingly, we found that RT followed a very similar profile then the subjective freedom of choice ratings (Figure S3). RT and freedom of choice indeed correlated on a trial-by-trial basis, revealing that trials with longer decision-times were associated with stronger feelings of free choice (see Supplementary Results 2.2).
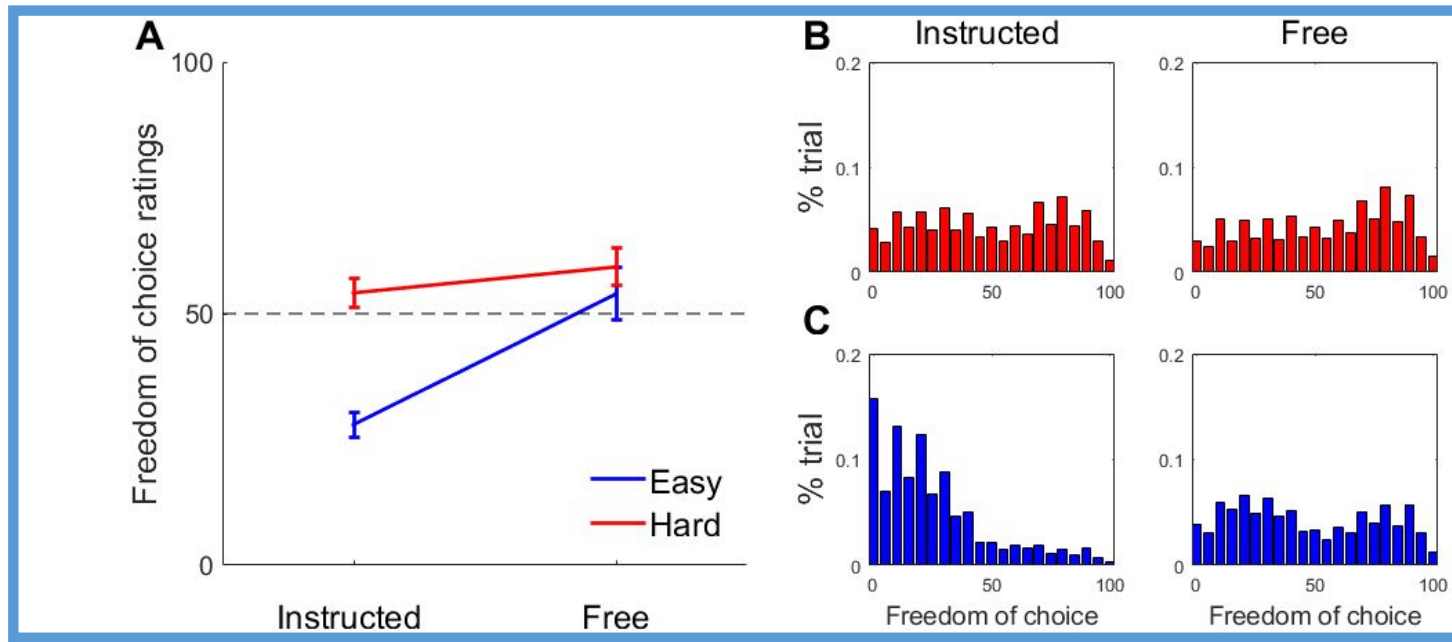

C
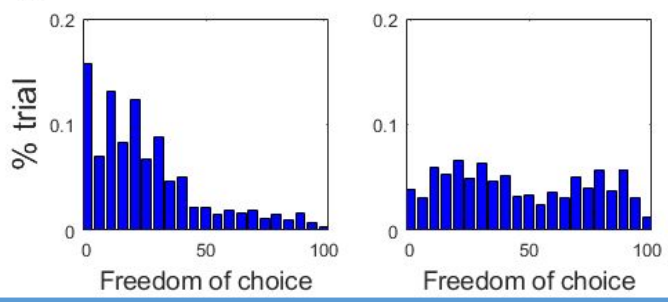

Figure 3: Subjective ratings of freedom of choice. A: Subjective ratings of freedom of choice averaged across participants for each difficulty and trial-type (Instructed versus Free) condition. B-C: Histograms of the freedom of choice ratings averaged across participants for each difficulty (row) and trial-type (column) condition. Error bars indicate standard error of the mean.

\subsection{Estimating objective freedom from stimulus-determination}

One of the first goal of the present experiment was to explore how much participants were actually influenced by random fluctuations in the exogenous perceptual signals when making "free" decisions. We used a reverse correlation method to measure objective freedom from stimulus-determination i.e., the extent to which the choice is independent of exogenous signals (Figure 2A, see Methods). This allowed us to estimate participants' behavioural sensitivity to visual information. A positive value of AUC indicated that the participant's choice was consistent with the stochastic fluctuations in stimulus motion while a negative value suggested that the choice opposed the direction of the signal fluctuations. 
262 Testing whether the stimulus had an entraining effect on the response in Free trials (Figure 4, left 263 column), we indeed found that the AUC was positive for both $\operatorname{Hard}(\mathrm{t}(13)=1.9, p=.040, d=0.51$, 264 one-tailed) and Easy conditions $(\mathrm{t}(13)=5.59, \mathrm{p}<.001, \mathrm{~d}=1.49$, one-tailed), showing that 265 participants' action choices were biased in the direction of the fluctuating motion stimuli. Moreover, 266 AUC was overall greater for easy than hard trials $(t(13)=\mathbf{2 . 6 5}, \mathbf{p}=\mathbf{. 0 2 0}, \mathbf{d}=\mathbf{0 . 7 1})$. This result suggests 267 that when faced with a clear cue to act freely, participants were more likely to base their decision on 268 sensory noise than when they were presented with an ambiguous sensory cue.

269 We investigated if the positive sensory biasing was still observed when participants reported high 270 or low subjective freedom of choice (Figure 4, middle and right columns). We split the freedom of 271 choice of scale by its midline and computed the AUC values separately for free trials associated with a 272 freedom of choice ratings below and above the midpoint of the scale. For low freedom of choice (Figure 2734 , middle column), we found that AUC was significantly higher than zero in Easy trials $(\mathrm{t}(13)=4.35$, $274 \mathrm{p}<.001, d=1.16$, one-tailed) but not for Hard trials $(\mathrm{t}(13)=\mathbf{0 . 7 8 1}, \mathrm{p}=. \mathbf{2 2}, \mathrm{d}=\mathbf{0 . 2 1}$, one-tailed) 275 resulting in a significant difference between the two $(t(13)=\mathbf{3 . 1 3}, p=\mathbf{. 0 0 8}, d=\mathbf{0 . 8 4})$. For high freedom 276 of choice ratings however (Figure 4, right column), the opposite pattern was found: AUC in easy 277 trials was not significantly different from $0(\mathrm{t}(\mathbf{1 3})=\mathbf{0 . 8 3}, \mathrm{p}=. \mathbf{2 1}, \mathbf{d}=\mathbf{0 . 2 2}$, one-tailed $)$ while it was 278 close to significance in hard trials $(t(13)=1.74, p=.053, d=0.47$, one-tailed). No significant 279 difference was observed between the two difficulty levels however $(t(13)=-\mathbf{0 . 6 3 1}, p=.54, d=-\mathbf{0 . 1 7})$.

280 Taken together, these results suggest that participants were able to track whether they were 281 influenced by the sensory fluctuations in the stimulus in the Easy but not in the Hard condition.

\section{Sensory Bias in Free trials}

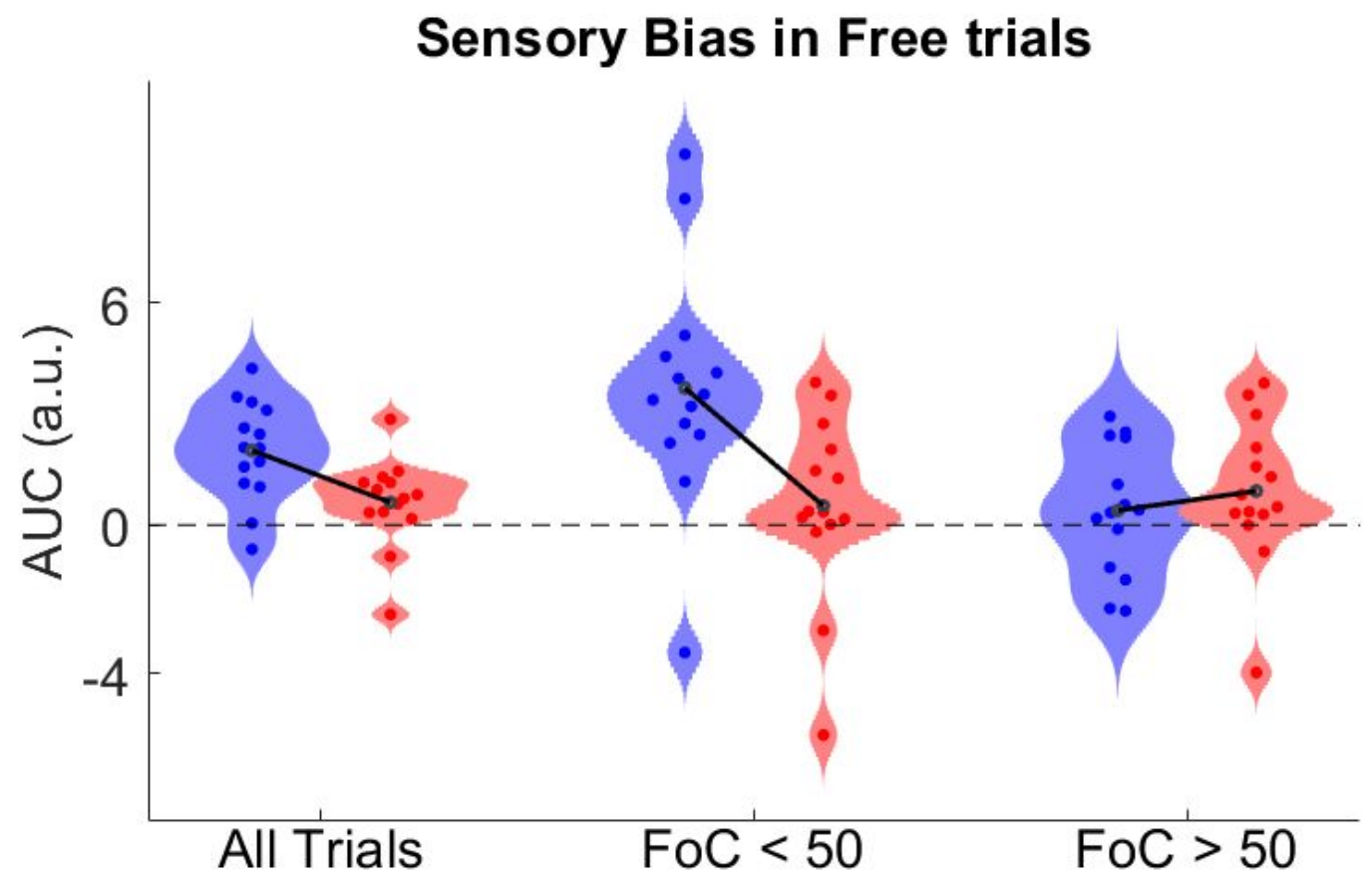


Figure 4: Objective freedom from stimulus-determination. AUC values averaged across participants for each difficulty (Easy: blue violin plots; Hard: red violin plots) considering all Free trials, Free trials with associated subjective rating inferior to 50 and Free trials with subjective ratings of Freedom of choice superior to 50. Null value of AUC indicate an absence of bias by sensory evidence (i.e. maximum objective freedom from stimulus-determination) and positive value indicate a positive bias towards sensory evidence (i.e. decreased objective freedom from stimulus-determination). Individual dots represent individual participants value and black dot represent group mean.

\subsection{Correlation between objective and subjective freedom of choice}

To confirm this result, we investigated further the correlation between the subjective reports of freedom of choice and the objective measure of how much participants were actually influenced by sensory information (Figure 5).

In each condition, we correlated our measure of objective freedom from stimulus-determination (trial-by-trial AUC values) with the subjective freedom of choice ratings for each condition including RT as an additional regressor using a linear mixed-model approach (see Supplementary Methods 1.4). Interestingly, we found a significant negative relation in easy free trials $(F(1,4862)=6.55, p=.011)$ suggesting that the more participants were influenced by sensory information, the less they felt free in their choice. However, this effect was not observed for hard trials $(F(1,4723)=0.169, p=.68)$. This result suggested that participants were able to introspect whether they were indeed influenced by the stimulus fluctuations in Easy but not in Hard trials.

If that was indeed the case, participant freedom of choice should reflect whether they were influenced by the stimulus fluctuations both when they choice by congruent with the direction and when on the contrary opposed them. To test that hypothesis, we separated trials according to the sign of the AUC (i.e., separately considering free-choice actions which followed or opposed the 'suggestion' of the random-dot motion stimulus respectively). We then performed the same regressions for each type of action separately. For hard trials, no significant correlation was found either for positive $(\mathrm{F}(1,2399)=0.0127, \mathrm{p}=.91)$ or negative AUC trials $(\mathrm{F}(1,2321)=0.109, \mathrm{p}=.74)$, as predicted by the absence of correlation when considering all trials together. Thus, we found no evidence for relation between AUC and subjective freedom of choice for Hard trials, whether participants followed the suggestion of the visual motion or not.

We then turned to Easy trials, for which previous results pointed towards a link between objective and subjective freedom of choice. Surprisingly, when considering positive AUC trials, in which participant responded congruently with the fluctuation of the evidence, no significant correlation was found with the subjective freedom of choice $(F(1,2573)=0.332, p=.56)$. When considering negative AUC trials, in which participants made a response opposing the direction of 
317 the evidence, a numerical trend toward a negative correlation was found $(F(1,2286)=2.24, p=$ 318 .13). Taken together, these results suggest that the previously observed correlation observed when 319 pooling positive and negative AUC may have been driven by a discrete difference in freedom of 320 choice between positive AUC trials (where participants responded congruently with the motion 321 evidence) and negative AUC trials (where participants responded incongruently with the motion 322 evidence) rather than a continuous relationship between subjective freedom of choice and AUC. 323 Consistent with this view, we found that in the easy condition, the mean subjective freedom of 324 choice was higher for negative AUC trials than for positive AUC trials $(t(13)=\mathbf{2 . 5}, p=.026, d=$ $3250.67)$ while this was not the case in the hard condition $(t(13)=-0.466, p=.65, d=-0.12)$.

326 Overall, these results suggest that subjective sense of freedom of choice increased when 327 participants choose the opposite response to the one favored by the stochastic fluctuation in the signal, 328 compared to when they made a response that matched the evidence in the sensory cue. In other 329 words, these results suggest an asymmetry in the sense of freedom of choice associated with 330 adhering or opposing the incoming sensory evidence, higher freedom being experienced in the 331 latter case.

332

A

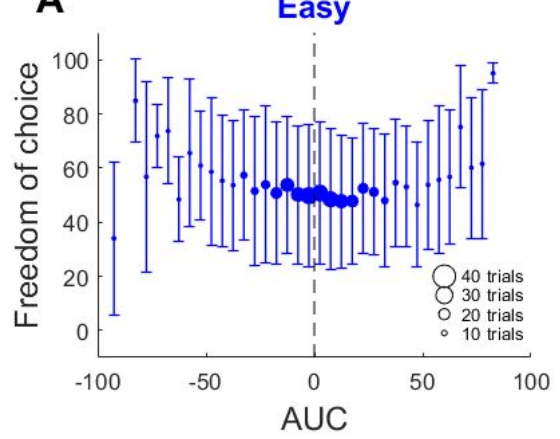

B

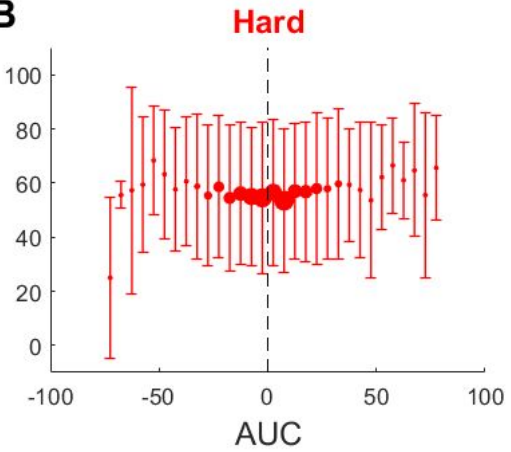

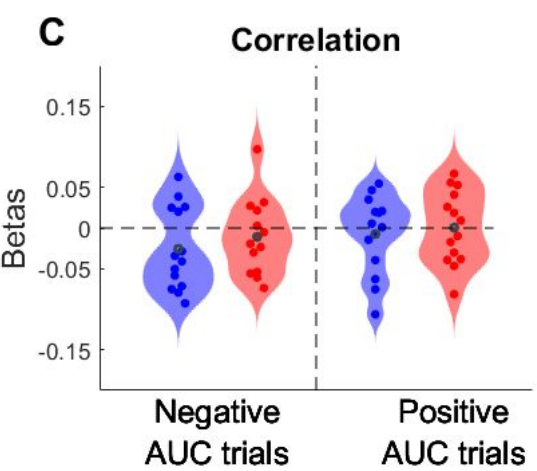

333

334 Figure 5: Relation between objective and subjective freedom from stimulus-determination. A-B: Scatter 335 plot of the distribution of subjective ratings of Freedom of Choice binned according to average AUC 336 values across participants for Easy (A) and Hard trials (B). Circle size reflects the average number of 337 trial across participants for each value of freedom of choice. Error bars reflect the standard deviation 338 of the subjective freedom of choice ratings across trials averaged across participants. C: Distribution 339 of the beta values for the correlation between AUC values and subjective ratings of freedom of choice 340 for each difficulty (Easy: blue violin plots; Hard: red violin plots) for trials with a positive AUC (left 341 of the middle line) and negative AUC (right of the middle line). Individual dots represent individual 342 participants value and black dot represent group mean 


\section{DISCUSSION (1303 WORDS)}

We report a novel investigation of perceived freedom of choice. Previous studies of free choice have mainly contrasted instructed actions (e.g., to left/right-pointing arrows) to endogenous response (e.g., double-headed arrow) (Kiesel et al., 2006; Le Bars et al., 2016; Wenke et al., 2010), controlling for the presence vs. absence of an endogenous component in action selection. In the present study we used a different approach, studying how momentary sensory evidence favoring left or right responses in the stimulus cueing free-choice trials ultimately influenced choice. We also manipulated the perceptual clarity of that cue. In an easy condition, participants could clearly identify when the stimulus required a free choice. Conversely, in the hard condition, participants had to select an action, without any clear perceptual marker of whether they were in a free or instructed context. This design therefore allowed us to vary orthogonally the free vs. instructed nature of choice, and the perceptual clarity of the action selection cue. We found that free choices were influences by instantaneous sensory information, even though the overall sensory evidence was designed to favour neither response. That is, participants apparently relied on external noise to break the symmetry between competing action alternatives.

Integration of exogenous and endogenous information seems uncontroversial for the 'hard' condition. In the hard condition, motion coherence was close to chance level for discriminating between free (ambiguous) and instructed (unambiguous) sensory cues. Participants might plausibly treat every hard trial as an instructed trial, and to try to detect the predominant dot motion direction. However, this explanation cannot explain the effect of sensory evidence on action choices in the easy condition, where sensory evidence was obviously ambiguous. In the easy condition, it was perceptually clear that participants should choose freely, rather than in response to the sensory evidence. Previous studies have demonstrated that free choices can be biased by external factors, including experimental manipulation such as subliminal priming (Eimer and Schlaghecken, 1998; Mattler and Palmer, 2012), stimulus repetition (Arrington and Logan, 2004; Demanet et al., 2013; Mayr and Bell, 2006) and other external factors such as previous responses patterns (Bode et al., 2014; Lages and Jaworska, 2012). We replicate some of these effects in the present study, showing that previous responses biased current action selection when little evidence was present to guide the current choice (see Supplementary Results 2.3). However, the present study goes beyond those results and show that noise fluctuations in sensory inputs can influence free choice.

Interestingly, signal fluctuations in external sensory information appeared to have a different impact on choice in easy compared to hard free-choice trials. In particular, the results suggested that 374 participants relied more on sensory evidence and were paradoxically less endogenous in easy trials, 375 when they knew that they were in a free decision context. While further studies will be needed to 376 determine precisely how the signal in each condition impacted the response, a plausible interpretation 377 rely on the clarity of the stimulus: a clear motion perception signal is more likely to be amplified than 
378 a weaker signal and used to break the symmetry between options when required to make an endogenous 379 choice. Interesting links can we made here with independent research on perception of motion showing 380 how the subjective experience of moving dots can vary non linearly with direction and motion coherence (Niwa and Ditterich, 2008; Ratcliff et al., 2018; Teodorescu et al., 2016).

One of the main goals of the present study was to investigate the sense of freedom of choice, only few studies having studied this subjective report (Filevich et al., 2013) and little being known beyond brain regions activated during these judgments. Interestingly, we found that participants experienced as much freedom when they were explicitly required to make a free choice than when no clear instruction on how to act was available. Although this result can appear somehow intuitive, it shows that explicit context might not influence the sense of freedom of choice as much as expected. We found that low freedom of choice ratings on easy trials were associated with strong influence of stimulus fluctuations, as might be expected. In contrast, when participants reported high freedom of choice ratings, no clear sensory biasing of their action choices was found. This result suggests that participants might be partially aware of whether their choices are endogenous or exogenous in origin, even when the exogenous signal is minimal, and has no systematic bias. A possible interpretation of this finding is that, given a clear instruction to make a free decision, participants searched for an external signal to break the symmetry between the two options. They then relied on exogenous sensory noise, in the same way that we sometimes decide to flip a coin to make a choice. Crucially, participants were able to introspect that they were doing so, and thus reported low freedom of choice. This "exploit sensory noise" strategy would fit with the view that free decisions are situations of high conflict and high choice difficulty (Nachev et al., 2005; Nachev and Husain, 2010) that require effortful processing. Exploiting sensory noise might thus provide an efficient way to decide between possible actions.

$400 \quad$ More detailed analysis showed that the ability to introspect the source of action decisions was, 401 however, limited. Trial-by-trial correlations between the strength of sensory influence and the 402 subjective freedom of choice appeared to be driven by a difference in reported freedom between 403 trials in which participants resisted the influence provided by stimulus fluctuations and the trials 404 where they adhered to it (Figure 5). Restricting analysis to the subset of trials where participants 405 indeed followed the evidence showed no influence on freedom of choice. Taken together, these results 406 suggest that when the stimulus is ambiguous, people would naturally tend to follow such evidence, even 407 when it is clear they need not do so. However, when they opposed the evidence, they appeared to 408 experience a greater sense of freedom. Perhaps unsurprisingly, this result suggests that the active 409 process of inhibiting external influences on action selection might play a role in subjective freedom 410 of choice. More interestingly, it suggests that people appear to mistake resisting exogenous influences 411 for being independent of them. This may qualify as a new type of 'metacognitive illusion'. Interestingly, 412 this view fits with the notion that the voluntary inhibition of actions might be tightly linked to 413 consciousness (Dehaene et al., 2003; Della Sala et al., 1991; Mayr, 2004). 
415 Our data speak against a strong dissociation between self-initiated and externally triggered actions 416 (cf. Schüür and Haggard, 2011). Our study shows that, even when internally-generated actions are 417 required, and are also actually experienced as such, some element of external driving may be present. 418 This view is compatible with recent work proposing that self-generated actions can be modelled as an 419 accumulation of evidence process similar to one used to account for other perceptual decisions 420 (Murakami et al., 2014; Schurger et al., 2012). We now suggest that free decisions reflect the 421 accumulation of both endogenous and exogenous signals. Crucially, our findings suggest the weight 422 given to each of these signals could change according to factors, such as the high vs. low availability of 423 information about the decision context, as in our easy vs. hard conditions respectively.

\subsection{Conclusion}

Our work offers a new way to investigate both the freedom of choice, and the balance between 426 endogenous and exogenous information in 'free' decisions. Using this method, we revealed a 427 dissociation between objective and subjective freedom of choice. When trying to produce a free action 428 we are still influenced by sensory information although we are not fully aware of such influence. We 429 further show that greater subjective experience of freedom arises from resistance to external influence, 430 rather than independence from it. The brain clearly has a capacity for endogenous generation of 431 information for action. However, the subjective experience of one's own voluntariness may not have 432 access to this generator. 
434 ACKNOWLEDGEMENT

435

436 This work was supported by a European Research Council Advanced Grant (HUMVOL, agreement 437 number 323943) to PH. We thank Ariel Zylberberg for providing code to compute motion energy and 438 for his helpful comments on RDK stimulus analysis. We thank Eugenia Kulakova and Nima 439 Khalighinejad for their useful comments and discussion. We thank Dennis Kuperberg for his help in $440 \quad$ statistical analysis.

\section{AUTHOR CONTRIBUTIONS}

443 L.C. contributed to designing the experiment, testing participants, analyzing data and writing the 444 manuscript. P.H. contributed to designing the experiment, analyzing data and writing the manuscript.

446 DECLARATION OF CONFLICTING INTERESTS

447 The authors declare there is no conflict of interests. 


\section{REFERENCES}

450

451

452

453

Arrington CM and Logan GD (2004) The cost of a voluntary task switch. Psychological Science 15(9): 610-615. DOI: 10.1111/j.0956-7976.2004.00728.x.

Bode S, He AH, Soon CS, et al. (2011) Tracking the Unconscious Generation of Free Decisions Using Uitra-High Field fMRI. PLoS ONE 6(6). DOI: 10.1371/journal.pone.0021612.

Bode S, Bogler C and Haynes J-D (2013) Similar neural mechanisms for perceptual guesses and free decisions. NeuroImage 65. Academic Press: 456-465. DOI: 10.1016/J.NEUROIMAGE.2012.09.064.

Bode S, Murawski C, Soon CS, et al. (2014) Demystifying "free will": The role of contextual information and evidence accumulation for predictive brain activity. Neuroscience \& Biobehavioral Reviews 47. Pergamon: 636-645. DOI: 10.1016/J.NEUBIOREV.2014.10.017.

Dehaene S, Artiges E, Naccache L, et al. (2003) Conscious and subliminal conflicts in normal subjects and patients with schizophrenia: The role of the anterior cingulate. Proceedings of the National Academy of Sciences 100(23): 13722-13727. DOI: 10.1073/pnas.2235214100.

Della Sala S, Marchetti C and Spinnler H (1991) Right-sided anarchic (alien) hand: A longitudinal study. Neuropsychologia 29(11): 1113-1127. DOI: 10.1016/0028-3932(91)90081-I.

Demanet J, De Baene W, Arrington CM, et al. (2013) Biasing free choices: The role of the rostral cingulate zone in intentional control. NeuroImage 72. Academic Press: 207-213. DOI: 10.1016/J.NEUROIMAGE.2013.01.052.

Eimer M and Schlaghecken F (1998) Effects of Masked Stimuli on Motor Activation: Behavioral and Electrophysiological Evidence. Journal of experimental psychology. Human perception and performance 24(6): 1737-1747. DOI: 10.1037/0096-1523.24.6.1737.

Filevich E, Vanneste P, Brass M, et al. (2013) Brain correlates of subjective freedom of choice. Consciousness and Cognition 22(4). Academic Press: 1271-1284. DOI: 10.1016/J.CONCOG.2013.08.011.

Hoffstaedter F, Grefkes C, Zilles K, et al. (2013) The 'what' and 'when' of self-initiated movements. Cerebral Cortex 23(3): 520-530. DOI: 10.1093/cercor/bhr391.

Kiesel A, Wagener A, Kunde W, et al. (2006) Unconscious manipulation of free choice in humans. Consciousness and Cognition 15(2): 397-408. DOI: 10.1016/j.concog.2005.10.002.

Lages M and Jaworska K (2012) How Predictable are "Spontaneous Decisions" and "Hidden 
Le Bars S, Hsu YF and Waszak F (2016) The impact of subliminal effect images in voluntary vs. stimulus-driven actions. Cognition 156: 6-15. DOI: 10.1016/j.cognition.2016.07.005.

Libet B, Gleason C a., Wright EW, et al. (1983) Time of Conscious Intention To Act in Relation To Onset of Cerebral Activity (Readiness-Potential). Brain 106(3): 623-642. DOI: 10.1093/brain/106.3.623.

Mattler U and Palmer S (2012) Time course of free-choice priming effects explained by a simple accumulator model. Cognition 123(3). Elsevier: 347-360. DOI: 10.1016/J.COGNITION.2012.03.002.

Mayr U (2004) Conflict, consciousness, and control. Trends in Cognitive Sciences 8(4): 145-148. DOI: 10.1016/j.tics.2004.02.006.

Mayr U and Bell T (2006) On how to be unpredictable: evidence from the voluntary task-switching paradigm. Psychol Sci 17(9): 774-780. DOI: 10.1111/j.1467-9280.2006.01781.x.

Murakami M, Vicente MI, Costa GM, et al. (2014) Neural antecedents of self-initiated actions in secondary motor cortex. Nature Neuroscience 17(11): 1574-1582. DOI: 10.1038/nn.3826.

Nachev P (2010) Free Choice and Voluntary Action. Psyche 16(1): 87-98.

Nachev P and Husain M (2010) Action and the fallacy of the 'internal': Comment on Passingham et al. Trends in Cognitive Sciences 14(5): 192-193. DOI: 10.1016/j.tics.2010.03.002.

Nachev P, Rees G, Parton A, et al. (2005) Volition and conflict in human medial frontal cortex. Current Biology 15(2). Elsevier: 122-128. DOI: 10.1016/j.cub.2005.01.006.

Niwa M and Ditterich J (2008) Perceptual decisions between multiple directions of visual motion. The Journal of neuroscience: the official journal of the Society for Neuroscience 28(17). Society for Neuroscience: 4435-45. DOI: 10.1523/JNEUROSCI.5564-07.2008.

Passingham RE, Bengtsson SL and Lau H (2010) Medial frontal cortex: from self-generated action to reflection on one's own performance. Trends in Cognitive Sciences 14(1). Elsevier Ltd: 16-21. DOI: 10.1016/j.tics.2009.11.001.

Plato (1987) Gorgias. Indianapol. Pub H (ed.).

Ratcliff R, Voskuilen C and Teodorescu A (2018) Modeling 2-alternative forced-choice tasks: Accounting for both magnitude and difference effects. Cognitive Psychology 103: 1-22. DOI: 
Schultze-Kraft M, Birman D, Rusconi M, et al. (2016) The point of no return in vetoing self-initiated movements. Proceedings of the National Academy of Sciences 113(4): 1080-1085. DOI: 10.1073/pnas.1513569112.

Schurger A, Sitt JD and Dehaene S (2012) An accumulator model for spontaneous neural activity prior to self-initiated movement. Proceedings of the National Academy of Sciences 109(42): E2904E2913. DOI: 10.1073/pnas.1210467109.

Schüür F and Haggard P (2011) What are self-generated actions? Consciousness and Cognition 20(4): 1697-1704. DOI: 10.1016/j.concog.2011.09.006.

Soon CS, Brass M, Heinze HJ, et al. (2008) Unconscious determinants of free decisions in the human brain. Nature Neuroscience 11(5): 543-545. DOI: 10.1038/nn.2112.

Soon CS, He AH, Bode S, et al. (2013) Predicting free choices for abstract intentions. Proceedings of the National Academy of Sciences 110(15): 6217-6222. DOI: 10.1073/pnas.1212218110.

Teodorescu AR, Moran R and Usher M (2016) Absolutely relative or relatively absolute: violations of value invariance in human decision making. Psychonomic Bulletin \& Review 23(1): 22-38. DOI: 10.3758/s13423-015-0858-8.

Wegner DM (2004) Précis of The illusion of conscious will. Behavioral and Brain Sciences 27(05). Cambridge University Press: 649-659. DOI: 10.1017/S0140525X04000159.

Wenke D, Fleming SM and Haggard P (2010) Subliminal priming of actions influences sense of control over effects of action. Cognition 115(1). Elsevier B.V.: 26-38. DOI: 10.1016/j.cognition.2009.10.016.

Wisniewski D, Goschke T and Haynes JD (2016) Similar coding of freely chosen and externally cued intentions in a fronto-parietal network. NeuroImage 134. Elsevier B.V.: 450-458. DOI: 10.1016/j.neuroimage.2016.04.044. 